\title{
A Construction of a Skewaffine Structure in Laguerre Geometry
}

by

\author{
Andrzej MATRAŚ
}

Presented by Jan RYCHLEWSKI

\begin{abstract}
Summary. J. Andre constructed a skewaffine structure as a group space of a normally transitive group. In this paper his construction is used to describe the structure of the set of circles not passing through a point of a Laguerre plane. Sufficient conditions to ensure that this structure is a skewaffine plane are given.
\end{abstract}

Introduction. The derived affine plane associated with a point $p$ of a Laguerre plane consists of all points nonparallel to $p$ and, as lines, all circles passing through $p$ (extended by all parallel classes not passing through $p$ ). A natural question is to characterize the structure of the set of circles not passing through $p$ by some linear geometry.

In the wide class of noncommutative (in general) linear structures constructed by J. Andre (cf. [1]) there are skewaffine planes which are good candidates for obtaining the characterization we are looking for. One of the classical examples of a skewaffine plane is the set of circles of the Euclidean plane with centers as basepoints (cf. [1]). Under weak conditions Wilbrink (cf. [12]) constructed, at a fixed point of a Minkowski plane, a skewaffine plane such that any straight line intersects any nonparallel line in exactly one point. This is known as a residual nearaffine plane. In the known examples (circles and hyperbolas) the construction is based on the observation that any circle (resp. hyperbola) has exactly one center which can be taken as the basepoint of the line corresponding to a given conic. This center is the image of our point $p$ in the symmetry with respect to the circle. In the case of Laguerre planes this construction cannot be used, since the image

2000 Mathematics Subject Classification: Primary 51B20.

Key words and phrases: miquelian Laguerre plane, symmetry axiom. 
of the point $p$ in the symmetry is parallel to $p$ and the symmetry does not distinguish any point which could be taken as a basepoint. It seems natural to investigate a symmetry with two pointwise fixed generators. However, two fixed generators do not define a symmetry of a Laguerre plane. To make the construction uniquely determined we fix an invariant pencil $\langle p, K\rangle$ of circles tangent at $p$. The basepoint of a line corresponding to a circle (which does not pass through $p$ ) is obtained as the point of tangency of the circle with the unique circle of the pencil $\langle p, K\rangle$. In contrast to Möbius and Minkowski planes, the basepoints belong to the corresponding circles, moreover, the residual skewaffine plane is not determined by the point $p$ alone, but by the pencil $\langle p, K\rangle$.

A large class of regular skewaffine planes was given in [1], [10] as the group space $\mathbf{V}(\mathbf{G})$ of a normally transitive group $\mathbf{G}$. In such skewaffine planes a line is obtained as the union of the basepoint and the orbit of another point with respect to the stabilizer of the basepoint.

The starting point of our paper is a group $\mathbf{G}$ of automorphisms of a Laguerre plane such that $\mathbf{V}(\mathbf{G})$ contains the set of circles not passing through $p$ as lines. The group $\mathbf{G}$ fixes points parallel to $p$ and the pencil $\langle p, K\rangle$. Minimal conditions for the group $\mathbf{G}$ are transitivity (call it (A1)) and circular transitivity for some circle of the pencil $\langle p, K\rangle$ (call it (A2)). The last axiom, (A3), is the condition that for any $L \in \mathcal{C}$ with $p \notin L$ there exists exactly one $M \in\langle p, K\rangle$ tangent to $L$. It guarantees that each circle not passing through $p$ corresponds to some line of $\mathbf{V}(\mathbf{G})$. We show that under axioms (A1)-(A3) the group $\mathbf{G}$ contains $\mathbb{L}$-translations and $\mathbb{L}$-strains fixing the pencil $\langle p, K\rangle$. This group is the subdirect product of the normal subgroup of $\mathbb{L}$-translations and an arbitrary subgroup of $\mathbb{L}$-strains with a fixed center (cf. Theorem 3.2). We give an example of a nonovoidal Laguerre plane which satisfies our axioms for some pencil $\langle p, K\rangle$ (cf. Remark 2.2). Miquelian Laguerre planes of characteristic distinct from 2 satisfy the axioms for any pencil (cf. Remark 2.1).

We use skewaffine planes to get a characterization of tangency in Laguerre planes. We obtain a condition determining whether a circle through two points tangent to a circle can be constructed (cf. Theorem 4.2). As an application we also show that the tangency points of the circles of pencils $\langle p, K\rangle$ and $\langle q, x\rangle$, where $q$ is parallel to $p$, form a circle (cf. Theorem 4.1).

Acknowledgements. The author wishes to thank the reviewer for many helpful suggestions.

1. Notations and basic definitions. A Laguerre plane is a structure $\mathbb{L}=(\mathcal{P}, \mathcal{C}, \sim)$, where $\mathcal{P}$ is a set of points denoted by small Latin letters, 
$\mathcal{C} \subset 2^{\mathcal{P}}$ is a set of circles denoted by capital Latin letters, and $\sim$ is an equivalence relation on $\mathcal{P}$ called parallelity (cf., for example, [4]).

(We use the notation $\sim$ to avoid confusion which would result from the use of the common notation "|l" for the parallelity of points of a Laguerre plane and of lines on a skewaffine plane.)

The equivalence classes of the relation $\sim$, called generators, are denoted by capital Latin letters. The following conditions must be satisfied:

(1) Three pairwise nonparallel points are joined by a unique circle.

(2) For every circle $K$ and any two nonparallel points $p \in K, q \notin K$ there is precisely one circle $L$ passing through $q$ which touches $K$ at $p$ (i.e. $K \cap L=\{p\}$ ).

(3) For any point $p$ and circle $K$ there exists exactly one point $q$ such that $p \sim q$ and $q \in K$.

(4) There exists a circle containing at least three points, but not all points.

The unique generator containing the point $p$ is denoted by $\bar{p}$. If $a, b, c$ are pairwise nonparallel points, the unique circle containing them is denoted by $(a, b, c)^{\circ}$. The circle tangent to a circle $K$ and passing through points $p, q$ $(p \in K, q \notin K, p \nsim q)$ is denoted by $(p, K, q)^{\circ}$. If $p \in K$, the symbol $\langle p, K\rangle$ stands for the pencil of circles tangent to $K$ at the point $p$. If $x, y$ are nonparallel, then the set of circles containing $x, y$ is called the pencil of circles with vertices $x, y$ and is denoted by $\langle x, y\rangle$. For a point $x$ and a circle $K$ the unique point of $K$ parallel to $x$ (which exists by (3)) is denoted by $x K$.

The derived plane at a point $p$ of a Laguerre plane $\mathbb{L}$, denoted by $\mathbb{A}_{p}$, consists of all points not parallel to $p$ and, as lines, all circles passing through $p$ (excluding $p$ ) and all generators not passing through $p$. This is an affine plane.

An automorphism of a Laguerre plane is a permutation of the set of points which maps circles to circles (and generators to generators). An automorphism $\phi$ is called central if there exists a point $p$ such that $\phi$ induces a central collineation of $\overline{\mathbb{A}}_{p}$, the projective extension of the derived affine plane $\mathbb{A}_{p}$.

An $\mathbb{L}$-translation is a central automorphism of a Laguerre plane $\mathbb{L}$ which fixes the points of $\bar{p}$ and induces a translation of $\mathbb{A}_{p}$ for some point $p$. The group of translations which fix the circles of a pencil $\langle p, K\rangle$ (respectively the family of generators $\mathcal{G})$ is denoted by $\mathbf{T}(\bar{p}, K)(\operatorname{resp} . \mathbf{T}(\bar{p}, \mathcal{G}))$ (cf. [6]).

An $\mathbb{L}$-strain with respect to a generator is a central automorphism of $\mathbb{L}$ which fixes the points of some generator $\bar{p}$ and the circles of some pencil $\langle q, M\rangle(p \nsim q)$. The group of all $\mathbb{L}$-strains fixing the points of $\bar{p}$ and the circles of the pencil $\langle q, M\rangle$ is denoted by $\Delta(\bar{p}, q, M)$. An involutory automorphism which fixes pointwise two generators $X, Y$ and a circle $M$ (not pointwise) is 
called a Laguerre symmetry and denoted by $\mathrm{S}_{X, Y ; M}\left(\mathrm{~S}_{X, Y ; M} \in \Delta(\bar{p}, q, M)\right.$ for $p \in X, q \in Y)$.

A group of central automorphisms is called circular transitive if the extension to $\overline{\mathbb{A}}_{p}$ of its restriction to $\mathbb{A}_{p}$ is linear transitive, i.e. is transitive on any line passing through the center. A group of automorphisms of $\mathbb{L}$ is called $\langle p, K\rangle$-transitive (resp. $\bar{p}$-transitive) if it contains a circular transitive group $\mathbf{T}(\bar{p}, K)$ (resp. $\mathbf{T}(\bar{p}, \mathcal{G})$, cf. [8]). A group of automorphisms of $\mathbb{L}$ is called $(\bar{p},\langle q, M\rangle)$-transitive if it contains a circular transitive group $\Delta(\bar{p}, q, M)$.

A skewaffine space (cf. [1]) is an incidence structure $\mathbb{S}=(X, \sqcup, \|)$, where $X$ is a nonempty set of points, denoted by small Latin letters, and

$$
\sqcup:\left\{(x, y) \in X^{2} \mid x \neq y\right\} \rightarrow 2^{X}
$$

is a function. Sets of the form $x \sqcup y(x \neq y)$ are called lines. They will also be denoted by capital Latin letters. The symbol \| denotes an equivalence relation among lines. The following axioms are assumed:

(L1) $x, y \in x \sqcup y$,

(L2) $z \in x \sqcup y \backslash\{x\}$ implies $x \sqcup y=x \sqcup z$ (exchange condition),

(P1) given any line $L$ and any point $x$ there exists exactly one line $x \sqcup y$ parallel to $L$ (Euclid's axiom),

(P2) $\forall x, x^{\prime}, y, y^{\prime}:\left(x \neq y, x^{\prime} \neq y^{\prime} \wedge x \sqcup y \| x^{\prime} \sqcup y^{\prime}\right) \rightarrow y \sqcup x \| y^{\prime} \sqcup x^{\prime}$ (symmetry condition),

(T) if $x, y, z$ are pairwise different points such that $x \sqcup y \| x^{\prime} \sqcup y^{\prime}$, then there exists a point $z^{\prime}$ such that $x \sqcup z \| x^{\prime} \sqcup z^{\prime}$ and $y \sqcup z \| y^{\prime} \sqcup z^{\prime}$ (Tamaschke's condition).

If we assume $x=x^{\prime}$ in axiom ( $\left.\mathrm{T}\right)$, then the axiom is called the affine Veblen condition (V).

We will consider additional conditions for a skewaffine space:

(Pgm) $\forall x, y, z \in X,\{x, y, x\}_{\neq} \exists w \in X$ with $x \sqcup y \| z \sqcup w$ and $x \sqcup z \| y \sqcup w$,

(Des) $\forall u, x, y, z, x^{\prime} \in X,\{u, x, y, z\}_{\neq} x^{\prime} \in u \sqcup x \backslash\{u\} \rightarrow \exists y^{\prime} \in u \sqcup y \backslash\{u\}$, $z^{\prime} \in u \sqcup z \backslash\{u\}$ with $x \sqcup y\left\|x^{\prime} \sqcup y^{\prime}, x \sqcup z\right\| x^{\prime} \sqcup z^{\prime}, y \sqcup z \| y^{\prime} \sqcup z^{\prime}$,

(Pap) $\forall u, x, y, z, x^{\prime} \in X,\left\{u, x, x^{\prime}\right\}_{\neq}$with $u \sqcup x=u \sqcup y=u \sqcup z \rightarrow$ $\exists y^{\prime}, z^{\prime} \in X$ with $u \sqcup x^{\prime}=u \sqcup y^{\prime}=u \sqcup z^{\prime}$ and $x \sqcup x^{\prime}\left\|z \sqcup z^{\prime}, x \sqcup y^{\prime}\right\|$ $y \sqcup z^{\prime}, y \sqcup x^{\prime} \| z \sqcup y^{\prime}$.

A skewaffine plane satisfying (Des) resp. (Pap) is called desarguesian resp. pappian.

If a line $L$ has the form $x \sqcup y$ then $x$ is called a basepoint of $L$. It is a simple consequence of the axioms that any line has either exactly one basepoint or all its points are basepoints (cf. [10]). A line such that all of its points are basepoints is called a straight line. A line which is not straight (and hence has exactly one basepoint) is called a proper line. 
A group $\mathbf{G}$ acting on a set $X$ is called normally transitive if $\mathbf{G}$ is transitive and $\mathbf{G}_{x} \backslash \mathbf{G}_{y} \neq \emptyset$ for any $x, y \in X$ with $x \neq y\left(\mathbf{G}_{x}\right.$ denotes the stabilizer of the point $x$ with respect to $\mathbf{G})$. For any group acting on a set $X$ one can construct a group space $\mathbf{V}(\mathbf{G})=(X, \sqcup, \|)$ with

- $x \sqcup y=\mathbf{G}_{x}\{x, y\}=\{x\} \cup \mathbf{G}_{x} y$,

- $L \| L^{\prime}$ if there exists $g \in \mathbf{G}$ such that $g L=L^{\prime}$ for any lines $L, L^{\prime}$.

The following theorem will be the basis of our construction $([1$, p. 5$]$, cf. also [10, Proposition 6.5, p. 94]).

TheOREM 1.1. The group space $\mathbf{V}(\mathbf{G})$ with respect to a normally transitive group $\mathbf{G}$ is a desarguesian skewaffine space.

A more detailed discussion of the properties of the group space $\mathbf{V}(\mathbf{G})$ can be found in [10].

EXAmPLE 1.1. Let $\mathbb{A}_{\mathbb{F}}=(\mathcal{P}, \mathcal{L}, \in)$ be an affine plane over a Euclidean field $\mathbb{F}$, and $\mathcal{L}_{0}:=\left\{L_{0, m} \mid m \in \mathbb{F}\right\}$, where $L_{0, m}:=\mathbb{F} \times m=\left\{(x, y) \in \mathbb{F}^{2} \mid\right.$ $y=m\}$. The set of affine transformations

$$
\mathbf{G}:=\left\{\varphi_{k, a, b} \mid k, a, b \in \mathbb{F}, k \neq 0\right\},
$$

where

$$
\varphi_{k, a, b}: \mathcal{P} \rightarrow \mathcal{P}, \quad \varphi_{k, a, b}(x, y):=\left(k x+a, k^{2} y+b\right),
$$

is a subgroup of Aut $\mathbb{A}_{\mathbb{F}}$ and $\mathbf{G}\left(\mathcal{L}_{0}\right)=\mathcal{L}_{0}$. A transformation $\varphi_{k, a, b}$ is a translation for $k=1$ and a strain for $k \neq 1$.

For any point $(a, b)$ the stabilizer $\mathbf{G}_{(a, b)}$ equals $\left\{\varphi_{k, a-k a, b-k^{2} b} \mid k \in \mathbb{F}^{*}\right\}$. For the group space $\mathbf{V}(\mathbf{G})=(\mathcal{P}, \sqcup, \|)$ we have

$$
(a, b) \sqcup\left(x_{0}, y_{0}\right):=\{(a, b)\} \cup\left\{(x, y) \mid y=r(x-a)^{2}+b\right\},
$$

where $r=\left(y_{0}-b\right) /\left(x_{0}-a\right)^{2}$ for $x_{0} \neq a$ and

$$
(a, b) \sqcup\left(x_{0}, y_{0}\right):=\{(a, b)\} \cup\left\{(x, y) \mid x=x_{0}, y=b+k^{2}\left(y_{0}-b\right)\right\}
$$

for $x_{0}=a$. The class of parallel parabolas of equation (1.1) is determined by the coefficient $r$. The class of parallel half-lines of equation (1.2) is determined by the sign of $y_{0}-b$. The set of straight lines is $\mathcal{L}_{0}$.

The construction can be generalized for any affine plane over a field of characteristic different from 2. The parallel classes of the sets of equation (1.2) are associated with the classes of squares of the field.

If the affine plane $\mathbb{A}_{\mathbb{F}}$ is the derived plane at a point $p$ of a miquelian Laguerre plane of characteristic different from 2, the lines of $\mathbf{V}(\mathbf{G})$ are associated with the circles of the Laguerre plane and subsets of the generators. The straight lines are associated with the line of one pencil of circles with vertex $p$. 
2. Residual skewaffine plane. Let $\langle p, K\rangle$ be a fixed pencil of circles of a Laguerre plane $\mathbb{L}=(\mathcal{P}, \mathcal{C}, \sim)$ and $\Delta(p, K)$ the group of automorphisms of $\mathbb{L}$ which fix the pencil $\langle p, K\rangle$ (not pointwise) and all points parallel to $p$. We assume that $\Delta(p, K)$ satisfies the following conditions:

(A1) $\Delta(p, K)$ is transitive on the set $\mathcal{P} \backslash \bar{p}$.

(A2) For any $x, y \in K \backslash\{p, r\}$ there exists $\sigma \in \Delta(p, K)_{r}$ with $\sigma(x)=y$. From (A1), (A2) we obtain a generalization of (A2).

Corollary 2.1. For any $x, y \in M \backslash\{p, r\}$ where $M \in\langle p, K\rangle$ there exists $\sigma \in \Delta(p, K)_{r}$ such that $\sigma(x)=y$.

Definition 2.1. The residual skewaffine plane with respect to $\langle p, K\rangle$ (written $\mathbb{S} A(p, K))$ is the group space $\mathbf{V}(\Delta(p, K))=(\mathcal{P} \backslash \bar{p}, \sqcup, \|)$.

By the definition $\Delta(p, K)$ is normally transitive and according to Theorem 2.1 we get

THEOREM 2.1. Suppose $\langle p, K\rangle$ is a fixed pencil of a Laguerre plane $\mathbb{L}=$ $(\mathcal{P}, \mathcal{C}, \sim)$ and conditions $(\mathrm{A} 1),(\mathrm{A} 2)$ are satisfied. Then the residual skewaffine plane $\mathbb{S} \mathbb{A}(p, K)$ is a skewaffine desarguesian space.

In the following let $\langle p, K\rangle$ be a fixed pencil such that the corresponding group $\Delta(p, K)$ satisfies axioms (A1), (A2).

PROPOSITION 2.1.

(a) If $r \sim x$, then $r \sqcup x=M \backslash \bar{p}$, where $M$ is a circle of $\langle p, K\rangle$ or a circle tangent at $r$ to some circle of $\langle p, K\rangle$.

(b) If $r-x$, then $r \sqcup x \subseteq \bar{r}$.

Proof. (a) If $r \notin K$, then consider the circle $L=(p, K, r)^{\circ}$. For $x \in L$, by Corollary 2.1, $L \backslash\{p\}=\Delta(p, K)_{r} x$. In the case $x \notin L$ we define $M=$ $(r, L, x)^{\circ}, x^{\prime}=x L$ and $y \in M \backslash \bar{p}, y \neq r$. The circle $M$ is invariant with respect to the group $\Delta(p, K)_{r}$ since it is tangent to $L$ at $r$ and has a fixed point at $\bar{p}$. By Corollary 2.1 there exists $\beta \in \Delta(p, K)_{r}$ such that $\beta\left(x^{\prime}\right)=y L$. Hence $\beta(x)=y$.

(b) This follows directly from the definition.

Definition 2.2. The line $x \sqcup y$ of $\mathbb{S} \mathbb{A}(p, K)$ is called special if $x \sim y$.

From the proof of Proposition 2.1 we get a further generalization of axiom (A2).

COROLlaRY 2.2. Let $r \notin \bar{p}$ and suppose $M$ is invariant with respect to $\Delta(p, K)_{r}$. Then for any $x, y \in M$ with $x, y \neq p M, r$ there exists $\sigma \in \Delta(p, K)_{r}$ such that $\sigma(x)=y$ (i.e. $\Delta(p, K)_{r}$ is circular transitive for any $\left.r \notin \bar{p}\right)$.

Proposition 2.2. The lines determined by the circles of the pencil $\langle p, K\rangle$ are straight lines. 
Proof. If $x, y$ are distinct points of a circle $L \in\langle p, K\rangle$, then $\Delta(p, K)_{x}(y)$ $=L \backslash\{p\}$ by Corollary 2.2.

The group $\Delta(p, K)$ fixes the generator $\bar{p}$ pointwise. Hence by the definition of parallelity we get:

Proposition 2.3. For any $M, N \in \mathcal{C}$ if $M \backslash \bar{p} \| N \backslash \bar{p}$, then $p M=p N$.

Propositions 2.1-2.3 provide a representation of the basic notions of a residual skewaffine plane of a Laguerre plane. However, this representation is not complete. The construction does not assume that any circle of the Laguerre plane corresponds to some line of the skewaffine plane. Similarly, the statements converse to Propositions 2.2 and 2.3 do not hold (as an example, take any miquelian Laguerre plane of characteristic 2). To make the representation more complete the following axiom will be needed throughout the rest of the paper.

(A3) For any circle $M$ such that $p \notin M$ there exists exactly one circle $L \in\langle p, K\rangle$ tangent to $M$.

Proposition 2.4. If $M$ is a circle such that $p \notin M$, then $M \backslash \bar{p}=x \sqcup y$, where $x$ is the point of tangency of $M$ with the unique circle of the pencil $\langle p, K\rangle$ and $y$ is any point of $M$ different from $x$ and $p M$.

Proof. According to (A3) there exists exactly one circle $L \in\langle p, K\rangle$ tangent to $M$. Let $x$ be the point of tangency. The circle $L$ is invariant with respect to $\Delta(p, K)_{x}$, since $L \in\langle p, K\rangle$ and $x \neq p$ is fixed. Hence $M$, as a circle tangent to an invariant circle and containing a fixed point $p M$, is invariant with respect to $\Delta(p, K)_{x}$. The assertion follows from Corollary 2.2.

According to Proposition 2.4 in the case $x \sqcup y=M \backslash \bar{p}$ the basepoint $x$ of the line $x \sqcup y$ will also be called the basepoint of the circle $M$.

Proposition 2.5. If $M \backslash \bar{p}$ is a straight line, then $M \in\langle p, K\rangle$.

Proof. Assume that $M \backslash \bar{p}=x \sqcup y=y \sqcup x$ for some distinct points $x, y \in M \backslash \bar{p}$ and $M \notin\langle p, K\rangle$. Then the subgroup of $\Delta(p, K)$ fixing the circle $M$ is transitive on its points, contrary to (A3).

Proposition 2.6. If $p M=p L$ with $p \notin M, L$, then $M \backslash \bar{p} \| L \backslash \bar{p}$.

Proof. From Proposition 2.4, $M \backslash \bar{p}=x \sqcup y$ where $x$ is the point of tangency of $M$ with some $M^{\prime} \in\langle p, K\rangle$ and $L \backslash \bar{p}=z \sqcup t$ where $z$ is the point of tangency of $L$ with some $L^{\prime} \in\langle p, K\rangle$. By (A1) there exists $\sigma \in \Delta(p, K)$ such that $\sigma(x)=z$. We obtain $\sigma\left(M^{\prime}\right)=L^{\prime}$ and hence $\sigma(M)=L$ by the touching axiom.

REMARK 2.1. In the case of miquelian planes of characteristic different from 2 any pencil $\langle p, K\rangle$ satisfies (A3) and the group $\Delta(p, K)$ has properties (A1) and (A2). Additionally, V $(\Delta(p, K))$ satisfies (Papp) and (Pgm). 
REMARK 2.2. Examples of nonmiquelian (even nonovoidal) planes satisfying (A1)-(A3) may be obtained if in the construction in [2] we put $f(x)=|x|^{r}(r>1)$ and $p=(\infty, 0), K=\{(x, 0) \mid x \in K\} \cup\{(\infty, 0)\}$. The transformations of the form $x^{\prime}=k x, y^{\prime}=|k|^{r} y$ (identity for points $(\infty, a))$ form the stabilizer of the point $(0,0)$.

REMARK 2.3. Axiom (A3) is satisfied for any pencil $\langle p, K\rangle$ of a topological Laguerre plane of dimension 2 and 4 (this is a special case of the solution of the Apollonius problem for such planes, cf. [11]).

\section{A characterization of the group $\Delta(p, K)$}

THEOREM 3.1. For any point $r \notin \bar{p}$ the stabilizer $\Delta(p, K)_{r}$ is a $(\bar{p},\langle r, L\rangle)$-transitive group of $\mathbb{L}$-strains $\Delta(\bar{p}, r, L)$ containing $\mathrm{S}_{\bar{r}, \bar{p} ; K}$, where $L=(p, K, r)^{\circ}$.

Proof. As in the proof of Proposition 2.4, for any $\phi \in \Delta(p, K)_{r}$ and $M \in\langle r, L\rangle$ we have $\phi(M)=M$. The $(\bar{p},\langle r, L\rangle)$-transitivity of the group $\Delta(p, K)$ follows from Corollary 2.2. To obtain $\mathrm{S}_{\bar{r}, \bar{p} ; K}$ consider an arbitrary $x \notin L \cup \bar{r} \cup \bar{p}$ and the circles $M=(r, L, x)^{\circ}, N=(p, L, x)^{\circ}$. According to (A3) these circles are not tangent, so there exists a point $y$ with $y \neq x$, $y \in M \cap N$. By $(\bar{p},\langle r, L\rangle)$-transitivity there exists $\psi \in \Delta(p, K)_{r}$ such that $\psi(x)=y$. Hence $\psi(N)=N$ and $\psi(y)=x$. This shows that $\psi=\mathrm{S}_{\bar{r}, \bar{p} ; K}$.

Proposition 3.1. The group $\Delta(p, K)$ is $\langle p, K\rangle$-transitive.

Proof. Let $x, y \in K$ be such that $\#\{x, y, p\}=3$. Consider an arbitrary circle $M$ such that $M \cap K=\{x, y\}$ and let $r$ be the basepoint of $M$. According to Theorem 3.1 the symmetries $\mathrm{S}_{\bar{r}, \bar{p} ; K}$ and $\mathrm{S}_{\bar{x}, \bar{p} ; K}$ exist and the superposition $\mathrm{S}_{\bar{r}, \bar{p} ; K} \circ \mathrm{S}_{\bar{x}, \bar{p} ; K}$ is a translation which maps $x$ to $y$.

From the proof of Proposition 3.1 we obtain:

Corollary 3.1. For any distinct points $x, y$ such that $x, y \neq p, x, y \in$ $R \in\langle p, K\rangle$ there exists $r \in R$ such that $\mathrm{S}_{\bar{r}, \bar{p} ; K}(x)=y$.

LEMma 3.1. Any fixed point free (outside $\bar{p}$ ) automorphism from $\Delta(p, K)$ is a translation.

Proof. Suppose there exists $x$ such that $x \nsim \phi(x)$ for some $\phi$ satisfying the assumptions of the lemma. Then $\phi(x) \nsim \phi^{2}(x)$. If $x \neq \phi^{2}(x)$, then the automorphism $\phi$ fixes the circle $M=\left(x, \phi(x), \phi^{2}(x)\right)^{\circ}$. If $x=\phi^{2}(x)$, then $\phi$ fixes any circle $M \in\langle x, \phi(x)\rangle$. Hence $p \in M$, because otherwise the basepoint of the circle $M$ is fixed. This means that $\phi$ is a translation with the invariant pencil $\langle p, M\rangle$. In the case $x \sim \phi(x)$ the assumption that there exists $y$ such $y \nsim \phi(y)$ implies that the circle $N=\left(y, \phi(y), \phi^{2}(y)\right)^{\circ}$ is invariant and $x N$ is fixed, a contradiction. Thus in this case $\phi$ is a translation which fixes all generators. 
Proposition 3.2. The group $\Delta(p, K)$ is $\bar{p}$-transitive.

Proof. Let $x \sim y, x \neq y$ and $r \nsim x$. Suppose $\phi \in \Delta(p, K)_{r}, z=\phi(x)$, $z^{\prime}=\mathrm{S}_{\bar{p}, \bar{r} ; K}(z)$ and $M=\left(y, z, z^{\prime}\right)^{\circ}$. Because $\mathrm{S}_{\bar{p}, \bar{r} ; K}(M)=M$ the basepoint $s$ of the circle $M$ is parallel to $r$. By Theorem 3.1 there exists $\psi \in \Delta(p, K)_{s}$ such that $\psi(z)=y$, so that $\psi \circ \phi(x)=y$. The automorphism $\psi \circ \phi$ fixes (not pointwise) two generators distinct from $\bar{p}$. Additionally it is not an $\mathbb{L}$-strain so it is fixed point free outside $\bar{p}$. By Lemma $3.1, \psi \circ \phi$ is a translation which maps $x$ to $y$.

The group of translations contained in $\Delta(p, K)$ will be denoted by $\mathbf{T}(p, \Delta)$.

THEOREM 3.2. Elements of the group $\Delta(p, K)$ without fixed points (outside $\bar{p})$ are either translations in the direction of any $L(p \in L)$ or translations which fix generators. The group $\mathbf{T}(p, \Delta)$ is transitive on the set $\mathcal{P} \backslash \bar{p}$ and $\mathbf{T}(p, \Delta) \unlhd \Delta(p, K)$. Elements of $\Delta(p, K)$ with fixed points are $\mathbb{L}$-strains and $\Delta(p, K) \simeq \mathbf{T}(p, \Delta) \rtimes \Delta(p, K)_{r}$ for any $r \notin \bar{p}$.

Proof. The first part of the theorem follows from Proposition 3.1, Proposition 3.2, and [7, Theorem 4.19, p. 100]. For any $\mathbb{L}$-strain $\phi$ and a translation $\psi$ the superposition $\phi \circ \psi \circ \phi^{-1}$ is a translation. Indeed, otherwise $x=\phi \circ \psi \circ \phi^{-1}(x)$ for some $x \notin \bar{p}$ and $\phi^{-1}(x)$ is a fixed point of the translation $\psi$, a contradiction.

Corollary 3.2. The group $\Delta(p, K)$ is of type $\mathbf{1 H}$ in the classification [8] of Laguerre planes.

Corollary 3.3. The group space $\mathbf{V}(\Delta(p, K))$ satisfies condition (Pgm).

Proof. The group $\mathbf{T}(p, \Delta)$ is commutative by [7, Theorem 4.14, p. 97] and transitive by $[7$, Theorem 4.19, p. 100]. Hence condition 5 in [10, Proposition 6.5, p. 94] is satisfied.

Corollary 3.4. Lines $A, B$ of a residual skewaffine plane are parallel iff there exists a translation $\phi$ such that $\phi(A)=B$.

\section{Some properties of residual skewaffine planes and their ap- plications to Laguerre planes}

Proposition 4.1. There are no three circles $L, M, N$ that are pairwise tangent at different points with $L \in(p, K)$ and $M \cap N \subset \bar{p}$.

Proof. Suppose the circles $M, N$ are tangent to the circle $L$ of the pencil $\langle p, K\rangle$ at points $x, y$ respectively and $M, N$ have a common point on the generator $\bar{p}$. By Corollary 3.1 there exists $r \in L \backslash \bar{p}$ such that $\mathrm{S}_{\bar{r}, \bar{p} ; K}(x)=y$. We obtain $\mathrm{S}_{\bar{r}, \bar{p} ; K}(M)=N$ and hence $r M=r N$ is another common point of $M, N$. 
Corollary 4.1. Parallel lines of $\mathbb{S} \mathbb{A}(p, K)$ determined by circles with basepoints on a straight line determined by a circle have a common point.

Proposition 4.2. Proper parallel lines of $\mathbb{S} \mathbb{A}(p, K)$ determined by circles are disjoint iff their basepoints are distinct and parallel.

Proof. $\Leftarrow$ Let $m, n$ be distinct and parallel basepoints of circles $M, N$ and suppose $M, N$ have a common point on the generator $\bar{p}$. A translation $\alpha \in \mathbf{T}(p, \mathcal{G})$ such that $\alpha(m)=n$ maps $M$ onto $N$, hence $(M \backslash \bar{p}) \cap(N \backslash \bar{p})=\emptyset$.

$\Rightarrow$ Assume the circles $M, N$ are tangent at a point $q \in \bar{p}$ and their basepoints $m, n$ are not parallel. Define $L=(p, K, m)^{\circ}, z=n L$ and $M^{\prime}=$ $(z, L, q)^{\circ}$. By Corollary $4.1, M^{\prime}$ is not tangent to $M$. This contradicts the part of the proposition already proved.

Proposition 4.3. Suppose that the basepoints of parallel lines $A, A^{\prime}$ belong to a straight line $B$ determined by a circle. If a straight line $C$ determined by a circle intersects $A$ then it intersects $A^{\prime}$.

Proof. Let $x, x^{\prime}$ be the basepoints of $A$ and $A^{\prime}$, respectively. Let also $y$ be one of the common points of $A, C$. There exists a translation $\tau \in \mathbf{T}(p, K)$ such that $\tau(x)=x^{\prime}$. We obtain $\tau(A)=A^{\prime}$ and the point $y^{\prime}=\tau(y)$ is a common point of $A^{\prime}, C$.

Lemma 4.1. Let circles $P, Q, R$ be tangent to a circle $L \in\langle p, K\rangle$ at pairwise distinct points. If $Q$ has common points with $P, R$, then $P, R$ have a common point.

Proof. If two of the circles $P, Q, R$ determine parallel lines, the assertion follows from Propositions 4.1 and 4.3. Assume $x \in P \cap Q, y \in Q \cap R$ and $x, y \notin \bar{p}$. If $Q \in\langle p, K\rangle$, consider a translation $\tau \in \mathbf{T}(p, K)$ such that $\tau(x)=y$, and the circle $P^{\prime}=\tau(P)$. We obtain $y \in P^{\prime} \cap R$ and $P^{\prime}$ is tangent to $L$. Hence, by the Veblen condition (V), the circles $P, R$ have a common point. In the case $Q \cap L=\{r\} \neq\{p\}$ instead of the translation $\tau$ we use the $\mathbb{L}$-strain $\phi \in \Delta(\bar{p}, K, r)$ such that $\phi(x)=y$, and the assertion follows by the Veblen condition or Proposition 4.3.

Definition 4.1. Let $L$ be a circle of the pencil $\langle p, K\rangle$. We say that points $a, b \notin L$ are equivalent (under $L$ ), and write $a \equiv_{L} b$, if $|P \cap Q| \geq 1$ for any circles $P, Q$ tangent to $L$ and passing through $a$ and $b$, respectively.

Lemma 4.1 gives the following.

Proposition 4.4. For any circle $L \in\langle p, K\rangle$ the relation $\equiv_{L}$ is an equivalence relation on the set $\mathcal{P} \backslash L$.

Proposition 4.5. For any points $a, b \in \mathcal{P} \backslash L, a \equiv_{L} b$ iff there exist circles $P, Q$ tangent to $L$ at distinct points and passing through $a, b$ respectively such that $|P \cap Q| \geq 1$. 
The set of points of a special line can be described by the relation $\equiv$ as follows:

Proposition 4.6. Let $x \sqcup y$ be a special line, and let $L=(p, K, x)^{\circ}$, $z \sim y, z \neq x$. Then $z \in x \sqcup y$ iff $z \equiv_{L} y$.

Proof. $\Rightarrow$ Assume $y^{\prime} \in(p, K, y)^{\circ}, M=\left(x, L, y^{\prime}\right)^{\circ}$ for some $y^{\prime} \neq p, y$. By the definition of $x \sqcup y$ there exists $\sigma \in \Delta(p, K)_{x}$ such that $\sigma(y)=z$. Then $\sigma(M)=M$. If $z^{\prime}=\sigma\left(y^{\prime}\right)$, we obtain $y \equiv_{L} y^{\prime} \equiv_{L} z^{\prime} \equiv_{L} z$.

$\Leftarrow$ Let $y \in N \in\langle p, K\rangle$ and $M$ be a circle passing through $z$ tangent to $L$ at a point different from $p$. Since $z \equiv_{L} y$, there exists a point $r$ such that $r \in M \cap N$. Define $P=(x, L, r)^{\circ}$ and $Q=(p, K, z)^{\circ}$. Then $P$ and $Q$ have a common point $s$ because $r \equiv_{L} z$. An $\mathbb{L}$-strain $\phi \in \Delta(\bar{p}, K, x)$ such that $\phi(r)=s$ maps $y$ to $z$.

Proposition 4.7. If $x \sim p, x \neq p$, then for any point $y \notin L, x \equiv_{L} y$ iff there exist exactly two circles $M, M^{\prime}$ tangent to $L$ such that $x, y \in M, M^{\prime}$.

Proof. It is sufficient to prove $\Rightarrow$. Let $N$ be any circle tangent to $L$ such that $x \in N$ and $P=(p, L, y)^{\circ}$. From $x \equiv_{L} y$ it follows that there exists $z \in P \cap N$. Then $M=\tau(N)$ where $\tau \in \mathbf{T}(p, K), \tau(z)=y$ and $M^{\prime}=S_{\bar{p}, \bar{y}, K}(M) \neq M$. Suppose, contrary to our claim, that there is a circle $M^{\prime \prime}$ through $x, y$, tangent to $L$ and distinct from $M, M^{\prime}$. Denote by $r, r^{\prime}, r^{\prime \prime}$ the basepoints of $M, M^{\prime}, M^{\prime \prime}$, respectively. There exists $\phi \in \Delta(p, K)_{r^{\prime \prime}}$ with $\phi(r)=r^{\prime}$. We have $\phi\left(M^{\prime \prime}\right)=M^{\prime \prime}, \phi(M)=M^{\prime}$ and $\phi(y) \neq x, y$. Hence $x, y, \phi(y)$ are three distinct points of two distinct circles $M^{\prime}, M^{\prime \prime}$, a contradiction.

Lemma 4.2. For any $q$ parallel to $p$ and different from $p$ there exists exactly one $q^{\prime}$ parallel to $p$ with the property:

$$
\forall x, y\left((x \nsim y, p \nsim x, y) \wedge q \in\left(x(p, K, x)^{\circ}, y\right)^{\circ} \rightarrow q^{\prime} \in\left(y,(p, K, y)^{\circ}, x\right)^{\circ}\right) .
$$

Proof. The assertion is a consequence of axiom (P2). If the point $q$ determines the class of lines parallel to a line $x \sqcup y$, then $q^{\prime}$ determines the class of lines parallel to $y \sqcup x$.

TheOREM 4.1. Let $q \neq p, q \sim p, x \nsim p$. Then the points of tangency of circles of the pencil $\langle p, K\rangle$ with circles of the pencil $\langle x, q\rangle$ form a circle (without a point of the generator $\bar{p}$ ).

Proof. Let $L=(p, K, x)^{\circ}$ and $M=(x, L, q)^{\circ}$. The point $x$ is the point of tangency of the circles $L$ and $M$ of the pencils $\langle p, K\rangle$ and $\langle q, x\rangle$, respectively. Consider an arbitrary circle $N \in\langle q, x\rangle, N \neq M$. By axiom (A3), there exists exactly one circle $P \in\langle p, K\rangle$ tangent to $N$ at some point $y$. The circle $Q=(x, L, y)^{\circ}$ is fixed by the group $\Delta(p, K)_{x}$. According to Corollary 2.2, any point of $Q$ distinct from $x$ and $p Q$ is the image of $y$ under some $\sigma \in$ $\Delta(p, K)_{x}$. Hence it is a point of tangency of circles of the pencils $\langle p, K\rangle$ and 
$\langle p Q, x\rangle$, respectively. It follows that the circle $Q$ satisfies the assertion of the theorem.

Corollary 4.2. The circle $Q$ determined in Theorem 4.1 passes through the point $q^{\prime}$ from Lemma 4.2 .

In the case of miquelian Laguerre planes of characteristic different from 2, the point $p$ in Theorem 4.1 can be chosen arbitrarily by Remark 2.1. For such planes we also obtain a condition determining whether a circle through two points tangent to a circle can be constructed.

THEOREM 4.2. Let $x, y$ be points and $L$ a circle with $x \nsim y, x, y \notin L$ of a miquelian Laguerre plane of characteristic distinct from 2. The following conditions are equivalent:

(1) There exist exactly two circles through $x, y$ tangent to $L$.

(2) Any circle through $x$ tangent to $L$ intersects any circle through $y$ tangent to $L$.

(3) There exist two intersecting circles tangent to $L$ at distinct points containing $x, y$, respectively.

Proof. According to Remark 2.1 the assertion follows by Definition 4.1, Proposition 4.5 and Proposition 4.7 applied to the pencil $\langle x L, L\rangle$.

REMARK 4.1. In miquelian Laguerre planes over a field $\mathbb{F}$ of characteristic different from 2 the conditions of Theorem 4.2 define the relation " $\equiv_{L}$ " for any circle $L$. In an analytic representation of such planes given in [9], for a circle $K=\{(x, 0) \mid x \in \mathbb{F}\} \cup\{(\infty)\}$, points $\left(a_{1}, b_{1}\right)$ and $\left(a_{2}, b_{2}\right)$ are equivalent with respect to $K$ iff $b_{2} \in b_{1} \mathbb{F}^{2}$. In this case the classes of parallelity of special lines correspond to the classes of squares of $\mathbb{F}$.

REMARK 4.2. If $\mathbb{F}$ is quadratically closed, then any special line coincides with a generator and is a straight line. In this case $\mathbb{S A}(p, K)$ contains two families of straight lines like a nearaffine residual plane connected with a Minkowski plane (cf. [12]). But the class of straight lines determined by the circles of the pencil $\langle p, K\rangle$ does not satisfy the condition of having exactly one common point with other lines.

\section{References}

[1] J. Andre, Introduction to noncommutative affine geometry, lectures held at Kuwait Univ., 1979.

[2] R. Artzy and H. Groh, Laguerre and Minkowski planes produced by dilatations, J. Geom. 26 (1986), 1-20.

[3] W. Benz, Vorlesungen über Geometrie der Algebren, Springer, Berlin, 1973.

[4] Y. Chen, A characterization of some geometries of chains, Canad. J. Math. 26 (1974), 257-272. 
[5] K. Grüning, Kegelschnitte und steinersche Ovale in Fano-Ebenen, Arch. Math. (Basel) 49 (1987), 459-464.

[6] E. Hartman, Transitivitätssätze für Laguerre-Ebenen, J. Geom. 18 (1982), 9-27.

[7] D. Hughes and F. Piper, Projective Planes, Springer, 1970.

[8] R. Kleinewillinghöfer, Eine Klassifikation der Laguerre-Ebenen nach $\mathcal{L}$-Streckungen und $\mathcal{L}$-Translationen, Arch. Math. (Basel) 34 (1980), 469-480.

[9] H. Makowiecka and A. Matraś, Central automorphisms of Laguerre planes, Demonstratio Math. 31 (1998), 753-763.

[10] J. Pfalzgraf, Über ein Modell für nichtkommutative geometrische Räume, Dissertation, Saarbrücken 1984.

[11] A. Schroth, Topological Circle Planes and Topological Quadrangles, Longman, 1995.

[12] H. A. Wilbrink, Nearaffine planes and Minkowski planes, Master's Thesis, Eindhoven Univ. of Technology, 1978.

\author{
Andrzej Matraś \\ Department of Mathematics and Informatics \\ UWM Olsztyn \\ Żołnierska 14 \\ 10-561 Olsztyn, Poland \\ E-mail: matras@uwm.edu.pl
}

Received January 30, 2006;

received in final form October 20, 2006 